\title{
EXPLORACIÓN DE RAÍCES SACRAS: INDICACIONES, TÉCNICA Y RESULTADOS
}

\author{
S. ARLANDIS GUZMÁN, J.M. ALAPONT ALACREU, M.Á. BONILLO GARCÍA, \\ J.L. RUIZ CERDÁ, E. MARTÍNEZ AGULLÓ, F. JIMÉNEZ CRUZ
}

\author{
Servicio de Urología. Hospital Universitario La Fe. Valencia.
}

Actas Urol Esp. 27 (7): 491-500, 2003

\section{RESUMEN}

EXPLORACIÓN DE RAÍCES SACRAS: INDICACIONES, TÉCNICA Y RESULTADOS

INTRODUCCIÓN: La neuromodulación mediante estimulación electrica de las raíces sacras constituye una alternativa terapéutica novedosa en el tratamiento de la disfunción miccional crónica (DMC). Pretende regular y devolver el equilibrio miccional alterado mediante impulsos eléctricos aplicados sobre las raíces sacras. La exploración de raíces sacras (ERS) es fundamental para considerar a un paciente candidato al implante definitivo del estimulador de la raíz sacra, comprobándose la integridad del arco reflejo sacro, la localización anatómica de la raíz y la respuesta sensitiva y motora del paciente.

PACIENTES Y MÉTODO: Entre enero de 2000 y noviembre de 2001 hemos efectuado un total de 43 ERS en 28 pacientes (13 hombres y 15 mujeres), con edades entre 22 y 76 años (edad media 56,3). Todos los pacientes habian sido diagnosticados de DMC con los siguientes sindromes clínicos: 13 Incontinencia Urinaria de Urgencia (IUU), 5 Síndrome de Frecuencia-Urgencia (SFU), 7 Disfunción de Vaciado (DV) y 3 cuadros mixtos (1 paciente SFU+DV y dos pacientes IUU+DV).

RESULTADOS: En las 43 ERS se obtuvo respuesta sensitivo y motora adecuada durante la fase aguda. En 8 ERS (18,6\%) se produjo una movilización precoz del electrodo que impidió la evaluación de la estimulación temporal. Las 35 ERS restantes (81,4\%) se evaluaron tras un periodo medio de estimulación de 6,7 días (3-15). No se produjeron complicaciones significativas y la exploración fue bien tolerada por todos los pacientes. Los resultados fueron satisfactorios ( $>50 \%$ de mejoría) en 7 pacientes $(53,8 \%)$ con IUU, 2 pacientes $(40 \%)$ con SFU, $1(14,2 \%)$ con DV y $1(33,3 \%)$ con sindrome mixto (IUU+DV). Así, del total de 28 pacientes con DMC sometidos a ERS, 11 han sido candidatos al implante del estimulador definitivo, lo que supone el $39,2 \%$ de los candidatos a esta terapia.

CONCLUSIONES: Actualmente la ERS se considera una prueba previa imprescindible para la implantación de un marcapasos urinario definitivo. Con ella seleccionamos a los pacientes en los que presuntamente este tratamiento ofrecerá buenos resultados. Es una técnica sencilla, bien tolerada y que se puede efectuar ambulatoriamente en cualquier centro.

PALABRAS CLAVE: Disfunción miccional. Neuromodulación sacra. Exploración de raíces sacras.

\section{ABSTRACT}

PERIPHERAL NERVE EVALUATION: INDICATIONS, TECHNIQUE AND RESULTS

INTRODUCTION: Sacral nerve neuromodulation is a new treatment modality for patients with chronic voiding dysfunction (CVD). The aim of this treatment is to restore the disbalanced micturition reflexes by electrical stimulation ot the sacral roots. Peripheral Nerve Evaluation (PNE) is the first step needed to determine wether sacral nerve stimulation for the control of lower urinary tract dysfunction is appropiate for a given patient, yielding accurate information about the location, integrity and function of sacral nerves, and motor and sensorial responses to electrical stimulation.

PATIENTS AND METHOD: 43 PNE in 28 patients (13 man and 15 women) were performed between january 2000 and november 2001 (mean age 56.3 years old, 22-76). Every patient have been diagnosed of CVD: 13 Urinary Urge Incontinence (UUI), 5 UrgencyFrequency Syndrome (UFS), 7 Dysfunctional Voiding (DV) and 3 Mixed Syndromes (1 patient UFS+DV and two patients with UUI+DV).

RESULTS: Every acute stimulation showed positive sensory and motor responses. There was premature lead migration in 8 PNE (18.6\%), preventing an appropiate evaluation of the temporal stimulation. 35 PNE were evaluated after a 6.5 days period (3-15) of electrical stimulation. There were no significative complications, and PNE was well tolerated. Good clinical results (>50\% reduction in symptoms) were observed in $7 / 13$ patients with UUI (53.8\%), $2 / 5$ patients with UFS (40\%), $1 / 7$ patients with DV (14.2\%) and $1 / 3$ patients with mixed syndromes (UUI+DV) (33.3\%). 11 of 28 patients with CVD (39.2\%) have been selected as candidates for permanent implantation.

CONCLUSION: Actually, PNE is the first step needed to determine wether sacral nerve stimulation for the control of lower urinary tract dysfunction is appropiate for a given patient. With PNE we can select properly adequate candidates for permanent implantation. It is an easy and well tolerated technique, and can be performed in any medical center in an outpatient basis.

KEY WORDS: Voiding dysfunction. Sacral neuromodulation. Peripheral nerve evaluation. 
$\mathrm{E}$ n la consulta del urólogo es frecuente encontrar pacientes con alteraciones miccionales de difícil diagnóstico y categorización. Habitualmente son personas con problemas miccionales de tipo crónico o larga evolución, en las que no se suele encontrar organicidad en las pruebas diagnósticas efectuadas y que ya han sido sometidas a múltiples tratamientos (la mayor parte de ellos con resultados poco satisfactorios).

Se conoce como Disfunción Miccional Crónica (DMC) a aquella condición que afecta a los órganos del tracto urinario inferior o a la musculatura del suelo pélvico y que altera el normal equilibrio de las distintas fases del ciclo miccional de forma crónica e independientemente de su etiología. Puede ser debida a causas neurológicas, musculares, orgánicas estructurales o funcionales (cuando no encontramos una causa de dicha alteración funcional con los medios diagnósticos disponibles a nuestro alcance).

La DMC engloba una serie de trastornos funcionales del tracto urinario inferior derivados de un funcionamiento alterado del sistema nervioso lo que origina unos reflejos vesicouretrales anormales, por lo que las manifestaciones clínicas de los pacientes con DMC son diversas, abarcando la incontinencia de urgencia, el sindrome frecuencia-urgencia y los síndromes retencionistas con disfunción de vaciado.

Desde un punto de vista clínico sintomático, la DMC tiene como expresión sindrómica las siguientes variantes reconocidas como candidatas a terapia mediante neuromodulación sacra:

1. Incontinencia Urinaria de Urgencia (IUU).

2. Disfunción de Vaciado (DV).

3. Sindrome frecuencia-urgencia (SFU).

4. Dolor pélvico crónico.

La neuromodulación mediante estimulación eléctrica de las raíces sacras constituye una alternativa terapéutica relativamente novedosa en nuestro país, no destructiva y reversible, para el tratamiento de los pacientes con DMC, en los que los tratamientos convencionales han resultado ineficaces o mal tolerados.

La neuromodulación consiste en la estimulación de vías nerviosas para modificar la actividad vesicoesfinteriana y la sensibilidad nociceptiva patológica mediante interacciones sinápticas. Existen dos formas de aplicación: mediante elec- trodos de superficie (neuromodulación periférica) o mediante electrodos que estimulan las raíces sacras, normalmente S3 (neuromodulación central). Con esta técnica se consigue modular el arco reflejo sacro patológico, recuperando su funcionamiento normal. Consiste básicamente en la colocación de un electrodo permanente en la proximidad de la tercera raíz sacra a través del foramen sacro (S3), que va conectado a un estimulador regulable por telemetría que se ubica subcutáneamente.

La exploración de raíces sacras (ERS) se realiza como paso previo a la colocación del estimulador definitivo. Es una exploración fundamental para considerar a un paciente como candidato al implante definitivo y con ella comprobamos la integridad del arco reflejo sacro, la localización anatómica de la raíz sacra y la respuesta sensitiva y motora que experimenta el paciente. Consta de dos fases: una primera fase (fase aguda) en la que implantamos el electrodo temporal de estimulación sacra y lo conectamos al estimulador externo, objetivando si existe respuesta sensitivomotora adecuada; y una segunda fase (prueba de estimulación temporal o fase subcrónica), que dura entre 3 y 7 días y en la que se observa si existe mejoría clínica significativa con el tratamiento.

Presentamos nuestra experiencia con la ERS como paso previo al implante del estimulador sacro definitivo.

\section{PACIENTES Y MÉTODO}

Efectuamos una revisión retrospectiva de las historias clínicas y de los formularios de registro de ERS (consensuados por el Grupo Español de Neuromodulación Sacra) de nuestros pacientes con DMC. Entre enero de 2000 y noviembre de 2001 efectuamos un total de 43 ERS en 28 pacientes ( 13 hombres y 15 mujeres), con edades comprendidas entre los 22 y 76 años (edad media 56,3 años). Realizamos una única exploración en 15 pacientes, dos exploraciones en 11 y tres en 2 pacientes.

Los criterios de selección fueron: edad mayor de 16 años, capacidad vesical mayor de $100 \mathrm{ml}$, ausencia de incontinencia de esfuerzo significativa, tracto urinario superior normal y diagnóstico de DMC refractaria a tratamientos convenciona- 
les. Incluimos a pacientes con clínica de incontinencia urinaria de urgencia (IUU), síndrome frecuencia urgencia (SFU), disfunción de vaciado (DV) y cuadros mixtos.

La historia clínica hizo hincapié en los síntomas urinarios de llenado y vaciado, inicio y duración de la sintomatología, antecedentes personales significativos (especialmente tratamientos previos sobre el tracto urinario inferior), caracterización de la incontinencia y presencia de síntomas y enfermedades asociadas. A todos los pacientes se les efectuó exploración física, analítica sanguínea, cultivo y sedimento de orina, ecografía urológica completa y estudio urodinámico (flujometría libre, medición de residuo post-miccional, cistomanometría de llenado y estudio presión-flujo). En casos seleccionados se completó el estudio del tracto urinario inferior con uretrocistografía y uretrocistoscopia con el fin de descartar patología orgánica. El estudio neurourológico se completó en algunos casos con estudio Electromiográfico específico, RMN de columna y Potenciales Evocados Somatosensoriales.

Excluimos a los pacientes con DMC en los que encontramos alguna contraindicación formal para la neuromodulación mediante estimulación eléctrica de raíces sacras (Tabla I).

Previamente a la ERS y durante el tiempo que dura la estimulación temporal el paciente debe rellenar un diario miccional, en el que anotará la hora y el volumen de cada micción, los episodios de incontinencia, el número de absorbentes utilizados, el volumen de líquido ingerido diariamente

\section{TABLA I}

CONTRAINDICACIONES PARA NEUROMODULACIÓN MEDIANTE EERS

\begin{tabular}{|c|}
\hline Incontinencia Urinaria Genuina de Esfuerzo \\
\hline ERS aguda y crónica deficiente \\
\hline Alteraciones anatómicas sacras \\
\hline Posibilidad de embarazo \\
\hline Síndrome de Reiter \\
\hline Diabetes Mellitus severa y/o neuropatía \\
\hline Patología médica severa concomitante \\
\hline Problemas psicológicos \\
\hline Infección urinaria activa \\
\hline
\end{tabular}

$\mathrm{y}$, los pacientes con dificultad de vaciado que estén sometidos a pauta de autosondaje, anotan el volumen residual de cada cateterismo. Con los datos del diario miccional objetivamos los cambios producidos con el tratamiento.

\section{DESCRIPCIÓN DE LA TÉCNICA DE EXPLORA-} CIÓN DE RAÍCES SACRAS

Consiste en la introducción a través del foramen sacro S3 de un electrodo que envía impulsos eléctricos a la raíz nerviosa S3 situada en vecindad. Estos impulsos son producidos por un generador impulsos eléctricos (GIE) externo regulable en cuanto a intensidad, anchura y frecuencia de pulso.

Con esta exploración comprobamos la integridad nerviosa del arco reflejo sacro, la localización anatómica de la raíz S3 y la respuesta motora y sensitiva del paciente. Consta de dos fases: una aguda o propiamente de exploración de raíces sacras y otra subcrónica o prueba temporal de estimulación eléctrica.

\section{Material necesario (Figs. 1A y B)}

1. Kit de prueba: incluye en un recipiente estéril todos los complementos para la prueba, como son paños de campo, povidona yodada, esponjillas para pintar la zona quirúrgica, rotulador, jeringa para anestesia local, aguja de insulina para infiltración, apósitos trasparentes, gasas, placas adhesivas de toma de tierra, dos agujas de foramen sacro (de $9 \mathrm{~cm}$ de longitud) y el electrodo de estimulación (microespiral siliconada con guía interna, que al retirarse mantiene la forma adquirida -efecto memoria-, garantizando una mínima tendencia a la movilización tras su inserción, con marcas radiológicas de profundidad).

2. GIE externo de prueba: proporciona el estímulo eléctrico necesario con la energía de una pila de $9 \mathrm{v}$, pudiendo fijar los parámetros de frecuencia $(\mathrm{Hz})$, duración del impulso (microsegundos), amplitud del impulso (voltios) y polaridad de los electrodos.

3. Cables de exploración: un cable largo para la fase aguda y uno corto que llevará el paciente durante los días de la estimulación temporal.

\section{Fase aguda (ERS)}

Se realiza de forma ambulatoria, con anestesia local. La posición del paciente es importante para conseguir que la superficie dorsal del hueso 

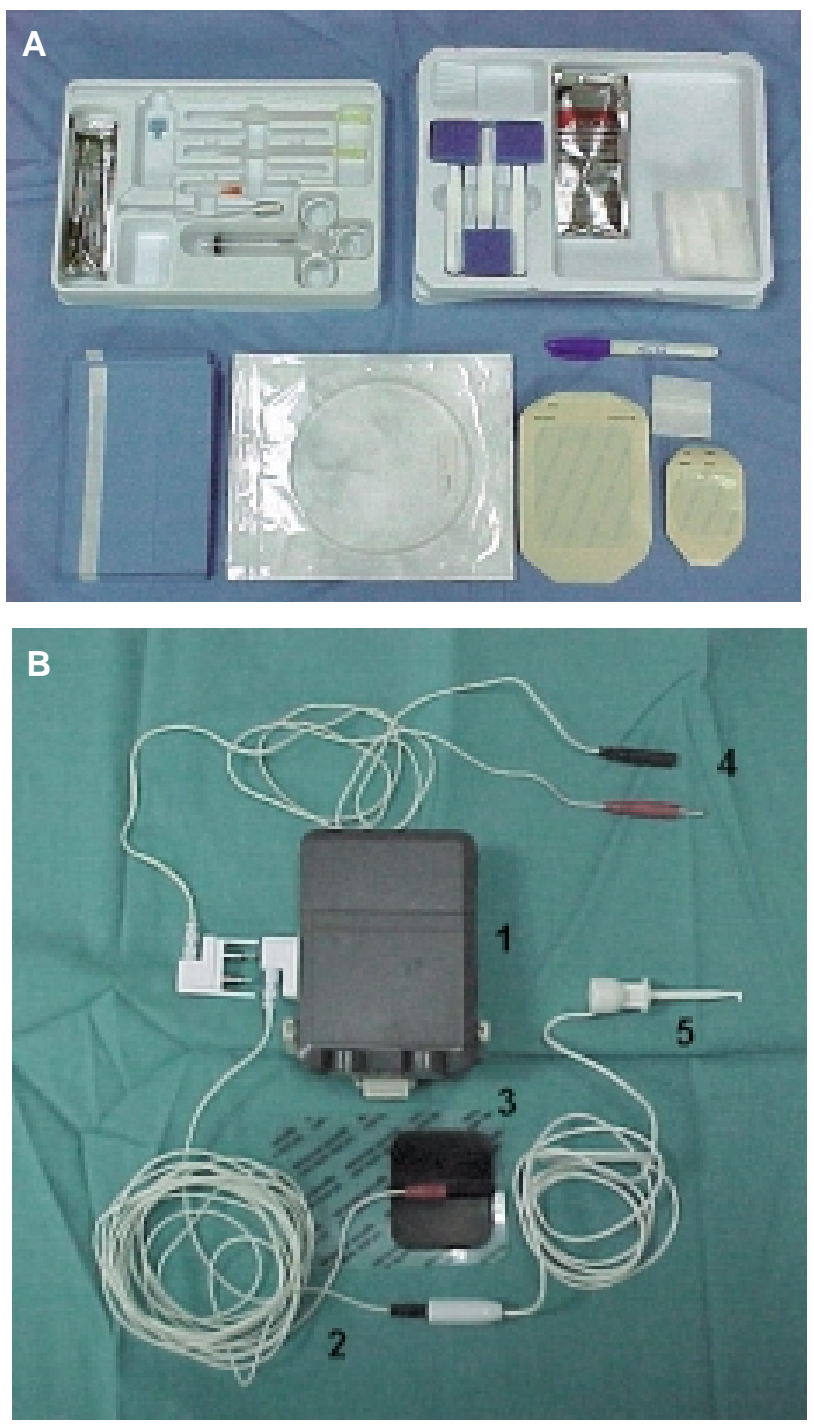

FIGURA 1. Material para la ERS.

A) Kit de exploración.

B) Estimulador externo (1), cable largo (2), toma de tierra (3), cable corto (4) y ganchillo de estimulación (5).

sacro quede horizontal, y el ángulo de entrada de las agujas de exploración sea el adecuado. Para ello se coloca en decúbito prono y con las caderas ligeramente flexionadas (unos $30^{\circ}$, consiguiéndose colocando una almohada bajo el pubis), lo que disminuye la lordosis lumbar, y con una discreta flexión de las piernas para que queden los pies elevados sin contactar con la superficie de la mesa. De este modo se pueden observar con detalle las respuestas motoras de las extremidades inferiores. Se colocan cintas adhesivas en los glúteos para separarlos y observar las respuestas motoras anales y del suelo pélvico (Fig. 2).

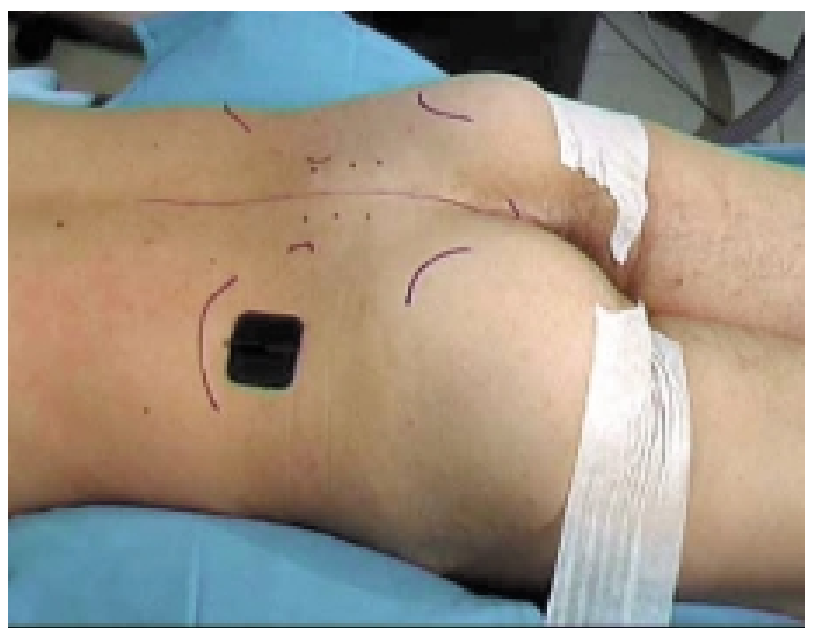

FIGURA 2. Posición y marcas óseas.

Se localizan las marcas óseas correspondientes al tercer foramen sacro, marcándose los orificios sacros y los accidentes óseos con rotulador. Esto se consigue mediante palpación de las siguientes referencias óseas: palas ilíacas, linea media de las apófisis espinosas sacras y lumbares, coxis, espinas ilíacas posterosuperiores y fosas isquiáticas. Aproximadamente un dedo a cada lado de la línea media sacra se localizan los agujeros sacros, separados unos de otros un dedo de altura aproximadamente. S2 se localiza aproximadamente a la altura de la línea que une las espinas ilíacas posteriores, S4 está a nivel de las fosas isquiáticas y S3 se sitúa a un dedo de altura entre cada una de ellas (Fig. 2). Existen varios métodos de localización basándose en estos accidentes óseos. En ocasiones puede ser útil la utilización de radioscopia para localizar estos accidentes anatómicos, aunque en la mayoría no es necesario.

Se administra anestésico local hasta llegar al hueso sacro. Es importante tener cuidado en no infiltrar anestésico directamente en el interior del foramen, ya que aboliriamos las respuestas motoras y sensitivas del estímulo eléctrico, dando la prueba un falso negativo.

Con la aguja de prueba se accede al foramen, siguiendo una dirección oblicua con el plano cutáneo de unos $60^{\circ}$ (aunque realmente es perpendicular a la superficie sacra) (Fig. 3). La aguja sólo trasmite la electricidad en el centímetro más distal y en la parte más proximal de ésta, estando el resto aislado eléctricamente. Así, aplicamos 


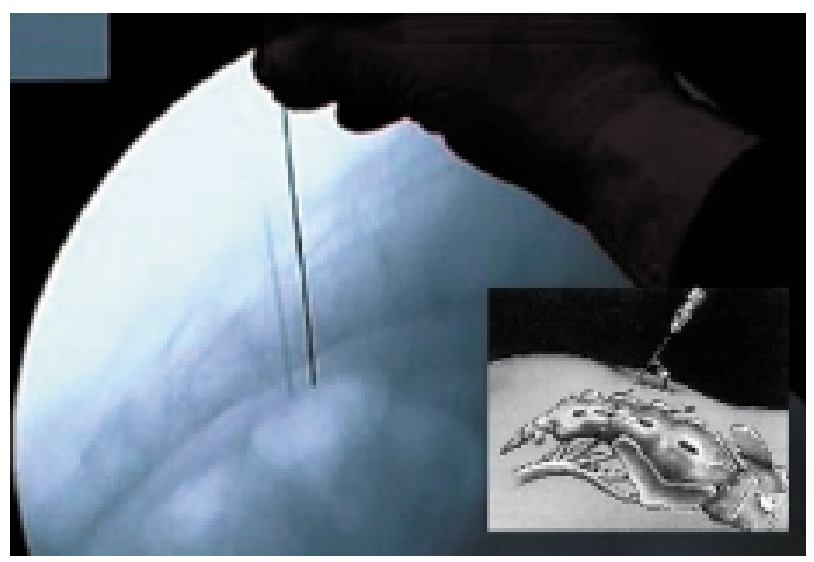

FIGURA 3. Rx lateral sacra mostrando la aguja introducida a través del foramen sacro $\mathbf{S 3}$.

el gancho de prueba en la parte más proximal, trasmitiendo el impulso a la parte distal, que está en contacto con la vecindad de la raíz sacra S3. La respuesta motora típica es una ligera flexión plantar del primer dedo del pie (a veces también del resto de dedos) y una elevación del periné y ano (por contracción del elevador del ano). La respuesta sensitiva consiste en una sensación de presión en recto y parestesias en escroto, pene, labios mayores o vagina. Es importante constatar esta respuesta motora y sensitiva, pues nos confirma la integridad nerviosa. Si no se consiguen estas respuestas, se abandona el procedimiento y el paciente no es candidato a la técnica. La estimulación de S2 o S4 dan respuestas motoras y sensitivas distintas que permiten distinguirlas de S3. Es preferible una buena respuesta a nivel del elevador del ano que plantar. Por otra parte, los movimientos y sensaciones deben notarse al mismo lado que se estimula eléctricamente. A veces es posible reproducir respuestas mixtas (S2-S3, o S3-S4), por variaciones anatómicas individuales en la inervación. Siguiendo el criterio de obtener preferentemente una buena respuesta del elevador del ano, puede ser necesario explorar otras raíces (S2 o S4) para conseguir respuestas adecuadas y ubicar el electrodo en el foramen correcto. Se inicia la estimulación con bajos voltajes $(0,5 \mathrm{v})$, incrementando progresivamente hasta obtener respuesta. Puede ser necesario variar la profundidad y el ángulo de penetración de la aguja, con el fin de lograr la posición idónea que produzca un mejor contacto con la raíz en el interior del foramen. Algunos autores se apoyan con registro electromiográfico de superficie simultáneo para evidenciar con mayor sensibilidad la respuesta muscular durante el estímulo. Si no se obtiene una respuesta adecuada tras 45-50 minutos, debe interrumpirse el proceso, y no se coloca el electrodo de prueba, siendo la prueba aguda negativa.

Una vez localizada la raíz y comprobada una adecuada respuesta muscular y sensitiva, se pasa a través del trócar de la aguja un electrodo especialmente diseñado para estimular la raíz durante los 5-7 días que durará la fase subcrónica o de estimulación temporal. Una vez comprobada una respuesta satisfactoria al estímulo, se retira con mucho cuidado la aguja, y a continuación la guía del electrodo. Se colocan apósitos trasparentes adhesivos sobre el electrodo y la zona de punción, procurando realizar un pequeño orificio en éste para que salga la sangre en caso de pequeña hemorragia (que no suele ser importante).

Este electrodo se conecta mediante el cable del paciente (más corto) al GIE externo, que se suele ajustar a $210 \mu \mathrm{s}, 20 \mathrm{~Hz}$ y 1-10 v, de modo continuo durante $24 \mathrm{~h}$ al día. $\mathrm{El}$ paciente puede variar la amplitud del impulso (entre 0 y 10 voltios, según el límite impuesto previamente por el médico).

Hay que insistir en que debe ajustarse el nivel de intensidad de la estimulación de forma que note su efecto en todo momento, sin llegar a ser dolorosa. Si el estímulo está por debajo del umbral sensitivo, éste no es efectivo.

\section{Fase subcrónica o prueba de estimulación temporal}

Tras la fase aguda, el paciente regresa a su domicilio y lleva a cabo sus actividades cotidianas, rellenando un diario miccional y una escala de dolor (si existe dolor asociado) durante los 3-7 días que dura el periodo de estimulación temporal. Pasados estos días se retira el electrodo y se compara el diario miccional con estimulación al basal. Si existe una mejoría en los parámetros miccionales superior al 50\%, el paciente se considera candidato para implante definitivo. De forma adicional se comprueba que tras la retirada del electrodo, los síntomas miccionales reaparecen, aunque en algunos pacientes persiste la 
mejoría clínica durante unas semanas tras la estimulación temporal. Esta no debe durar más de 21 días, ya que rebasado este tiempo pueden existir problemas en su extracción, pudiendo romperse y quedar parcialmente alojado en el foramen.

Es muy importante preguntar al paciente antes de retirar el electrodo dónde nota la sensación del estímulo, pues podemos encontrarnos con falsos negativos debidos a una mala técnica o complicaciones durante la estimulación temporal.

\section{RESULTADOS}

El tipo de DMC que presentaban los 28 pacientes fue: 13 Incontinencia Urinaria de Urgencia (IUU), 5 Síndrome de FrecuenciaUrgencia (SFU), 7 Disfunción de Vaciado (DV) y 3 cuadros mixtos (1 paciente SFU+DV y dos pacientes IUU+DV). El tiempo de evolución de la sintomatología fue de 3 meses a 18 años (media 4,7 años).

En los pacientes con IUU el número de escapes diarios fue entre 1 y 2 en 5 pacientes, entre 3 y 5 en 4 y más de 6 escapes en otros 6 pacientes. Tres pacientes no utilizaban absorbentes y el resto entre 3 y 6 absorbentes diarios. El grado de escape era leve en 4 pacientes, moderado en $5 \mathrm{y}$ severo en 4.

En los pacientes con SFU la frecuencia miccional diurna osciló entre 8 y 24 micciones (mediana 14) y la nocturna entre 3 y 6 (media 4).

Los pacientes con DV, 2 se autosondaban 2 veces al día, 2 pacientes $3 /$ día y 1 paciente 4 /día, oscilando los residuos entre 100 y $400 \mathrm{ml}$, mientras que 2 pacientes tenían clínica de dificultad miccional pero no se autosondaban.

Los hallazgos urodinámicos más característicos están reflejados en la Tabla II.

\section{TABLA II}

HALLAZGOS URODINÁMICOS

\begin{tabular}{||l|c|}
\hline Perfil urodinámico & № pacientes \\
\hline Detrusor hiperactivo & 15 \\
\hline Urgencia sensorial & 4 \\
\hline Detrusor hipoactivo & 5 \\
\hline Síndrome de Fowler & 1 \\
\hline Micción no coordinada & 1 \\
\hline Disinergia vésico-esfinteriana & 2 \\
\hline
\end{tabular}

Efectuamos un total de 43 ERS en 28 pacientes. Efectuamos una sola exploración en 15 pacientes, dos ERS en 11 y hasta 3 en 2 pacientes. En la mayoría de las exploraciones (37 ERS) ubicamos un único electrodo de estimulación, pero en 6 ocasiones dejamos colocados 2 electrodos ya que se trataba de pacientes en los que se había producido una movilización del electrodo en una ERS previa, o bien el efecto terapéutico había sido limítrofe. La ubicación de los electrodos en las 43 ERS fue la siguiente:

- 6 ERS con doble electrodo: 5 S3 bilateral, 1 S3 dcha + S4 dcha.

- 37 ERS con electrodo simple: 18 S3 dcha, 17 S3 izquierda, 1 S4 dcha y 1 S4 izda.

Las ubicaciones en raíz S4 se deben a que encontramos una mejor respuesta neuromuscular a este nivel. En esta primera fase conseguimos respuestas sensitivo-motoras en todos los pacientes, estimulando entre 1 y 2 voltios en la mayoría de los casos (Fig. 4). No se produjeron complicaciones en esta fase de la exploración.

De las 43 ERS efectuadas (fase aguda), 8 $(18,6 \%)$ consultaron antes de que terminara el periodo de tiempo necesario para evaluar la respuesta clínica, debido a que habían perdido el estímulo sobre la raíz sacra por movilización pre$\mathrm{coz}$ del electrodo. Hay que destacar que $7 \mathrm{de}$ estos casos ocurrieron cuando utilizábamos los electrodos antiguos, que tenían una mayor tendencia a la movilización en comparación con los de nuevo diseño. En 35 (81,4\%) ERS sí pudimos evaluar la respuesta terapéutica tras un periodo medio de 6.7 días (3-15) desde la implantación del electrodo.

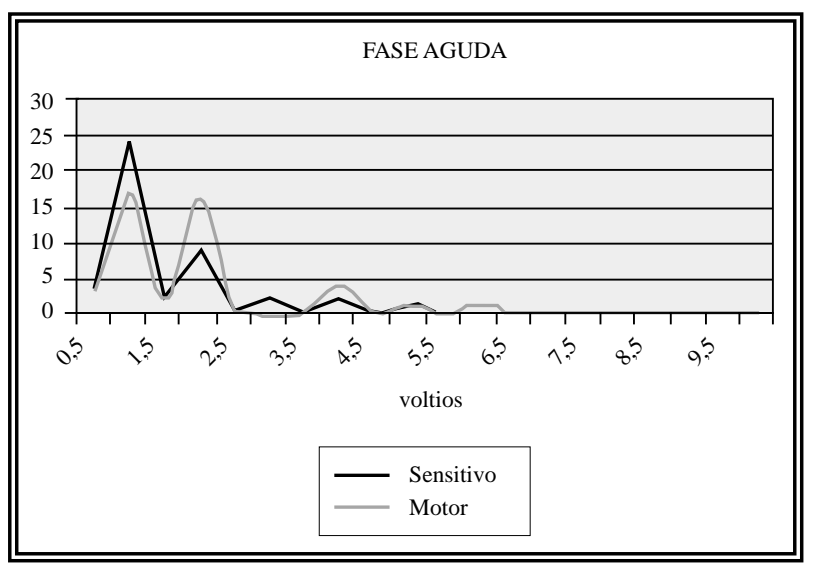

FIGURA 4. Umbrales de estimulación en la fase aguda. 
Previamente a la retirada del electrodo evaluamos de nuevo el umbral de respuesta sensitivomotora, observando que éste había aumentado en la mayoría de los casos (Fig. 5). Esto lo atribuimos por un lado a un fenómeno de acomodación nerviosa al estímulo eléctrico, y por otro a pequeñas movilizaciones del electrodo que hacen que la estimulación no se produzca en la zona óptima.

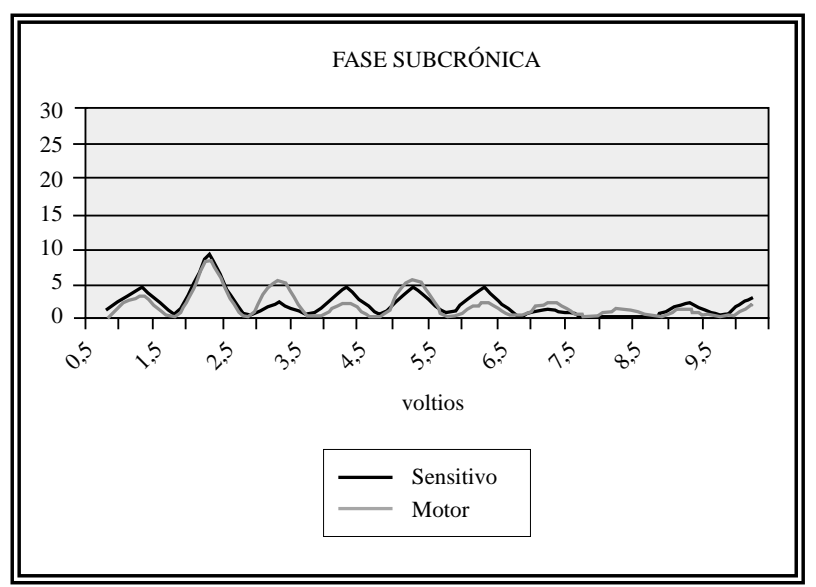

FIGURA 5. Umbrales de estimulación en la fase subcrónica.
Tras entrevistar al paciente y evaluar los diarios miccionales valoramos la mejoría clínica, el grado de satisfacción subjetiva (escala de 1 a 10 , de menor a mayor satisfacción) y el efecto terapéutico (0-50\%: mejoría no significativa; 50-90\%: mejoría significativa; 100\%: mejoría completa). Los resultados fueron satisfactorios $(>50 \%$ de mejoría) en 7 pacientes $(53,8 \%)$ con IUU, 2 pacientes $(40 \%)$ con SFU, $1(14,2 \%)$ con DV y 1 $(33,3 \%)$ con síndrome mixto (IUU+DV) (Tabla III).

En los pacientes con IUU que mejoraron, todos estaban secos excepto una paciente que pasó de tener 6 escapes moderados diarios a uno diario leve. El grado subjetivo de satisfacción fue de 9,4 (8-10). En el resto no se produjo ninguna mejoría, excepto una paciente que pasó de 10 a 3 escapes diarios, aunque la severidad de los mismos no varió, produciéndose efectos secundarios a la estimulación (dolor anal y debilidad intensa en la pierna ipsilateral) que disminuyeron el grado de satisfacción subjetivo percibido por aquella (puntuación = 1). El grado subjetivo de satisfacción en el grupo que no mejoró fue de 2,3 (1-6).

TABLA III

RESULTADOS EN LOS PACIENTES CANDIDATOS A IMPLANTE DE MARCAPASOS URINARIO DEFINITIVO

\begin{tabular}{|c|c|c|c|c|c|c|}
\hline \multirow[t]{2}{*}{ Grupo } & \multirow[t]{2}{*}{ Paciente } & \multirow[t]{2}{*}{ Sexo } & \multicolumn{2}{|c|}{ Clínica } & \multirow{3}{*}{$\begin{array}{c}\text { Efecto } \\
\text { terapéutico } \\
\text { (\%) }\end{array}$} & \multirow{3}{*}{$\begin{array}{c}\text { Grado } \\
\text { satisfacción } \\
\text { subjetiva } \\
(1-10)\end{array}$} \\
\hline & & & Antes & Después & & \\
\hline \multirow{8}{*}{$\begin{array}{l}\text { IUU* } \\
(7 / 13)\end{array}$} & & & \multicolumn{2}{|c|}{$N^{o}$ escapes } & & \\
\hline & AMGS & $\mathrm{F}$ & 6 & 1 & $50-90$ & 10 \\
\hline & MPM & $\mathrm{F}$ & 6 & 0 & $50-90$ & 9 \\
\hline & ASG & $\mathrm{F}$ & 3 & 0 & $90-100$ & 10 \\
\hline & AMN & $\mathrm{F}$ & 4 & 0 & $90-100$ & 8 \\
\hline & RDT & $\mathrm{M}$ & 1 & 0 & $50-90$ & 9 \\
\hline & ASS & $\mathrm{M}$ & 1 & 0 & $90-100$ & 10 \\
\hline & JVC & $\mathrm{M}$ & 3 & 0 & $90-100$ & 10 \\
\hline \multirow{3}{*}{$\begin{array}{l}\text { SFU* } \\
(2 / 5)\end{array}$} & & & \multicolumn{2}{|c|}{$F M D / F M N^{* *}$} & & \\
\hline & JMMR & M & $24 / 6$ & $11 / 6$ & $50-90$ & 5 \\
\hline & EMT & $\mathrm{M}$ & $16 / 6$ & $6-5$ & $50-90$ & 8 \\
\hline \multirow{2}{*}{$\begin{array}{l}\text { DV* } \\
(1 / 7)\end{array}$} & & & \multicolumn{2}{|c|}{ Cateterismos } & & \\
\hline & PRT & $\mathrm{F}$ & 4 & 0 & $90-100$ & 10 \\
\hline \multirow{4}{*}{$\begin{array}{l}\text { Sdr. Mixto } \\
\text { (IUU+DV) } \\
(1 / 3)\end{array}$} & & & \multicolumn{2}{|c|}{$N^{o}$ escapes } & & \\
\hline & BHR & $\mathrm{F}$ & 6 & 2 & \multirow[t]{3}{*}{$50-90$} & \multirow[t]{3}{*}{10} \\
\hline & & & \multicolumn{2}{|c|}{ Catererismos } & & \\
\hline & & & 3 & 0 & & \\
\hline
\end{tabular}

*IUU: incontinencia urinaria de urgenica; SFU: síndrome frecuencia-urgencia.

DV: dificultad para el vaciadovesical; el caso mixto corresponde a IUU+DV.

**FMD: frecuencia miccional diurna; FMN: frecuencia micional nocturna. 
Los dos pacientes con SFU que mejoraron pasaron de 16 y 24 micciones diarias a 6 y 11 respectivamente, mientras que la nicturia pasó de 6 y 11 a 5 y 6 .

La paciente con DV que mejoró estaba en retención completa, practicándose 4 cateterismos diarios, pasando tras la estimulación temporal a micciones completas sin residuo.

Una paciente con síndrome mixto (IUU+DV) pasó de 6 escapes moderados diarios con el uso de 5 absorbentes a 2 escapes leves con 2 absorbentes diarios. Por otra parte, se cateterizaba 3 veces al día, con residuos medios de $150 \mathrm{ml}$, pasando tras la estimulación temporal a no precisar cateterismos (ausencia de residuo postmiccional).

Así pues, del total de 28 pacientes con DMC sometidos a ERS, 11 han sido seleccionados para implantar el estimulador definitivo, lo que supone el $39,2 \%$ de los candidatos a esta terapia.

\section{DISCUSIÓN}

La estimulación eléctrica de raíces sacras es una alternativa terapéutica no destructiva y reversible indicada en pacientes con DMC en los que los tratamientos conservadores convencionales han resultado ineficaces o mal tolerados.

El tratamiento de la DMC debe comenzarse con las medidas terapeúticas iniciales (higiénicodietéticas, terapia conductual y farmacológica). Como segundo escalón podemos emplear electroestimulación periférica, cinesiterapia pelviana y biofeedback. Cuando fracasan estas medidas planteamos al paciente las técnicas de neuromodulación central mediante implante de marcapasos urinario definitivo como paso previo a soluciones quirúrgicas más agresivas que implican alteraciones anatómicas irreversibles en el tracto urinario inferior, como son la cistoplastia de aumento o la derivación urinaria. En la elección del tratamiento también debemos tener en cuenta las características físicas, funcionales, psíquicas y sociales del enfermo, así como su propia voluntad.

La ERS es una prueba previa imprescindible a la implantación de un marcapasos urinario definitivo. Es relativamente fácil de realizar, bien tolerada con anestesia local y no precisa ingreso hospitalario del paciente. Reproduce los efectos del implante definitivo y, si existe mejoría sintomatológica, permite conocer a priori el resultado del implante definitivo (factor pronóstico de respuesta al tratamiento: selecciona a los pacientes en los que presuntamente se obtendrán buenos resultados). De esta forma, tanto el médico como el paciente pueden tomar la decisión de continuar sobre una respuesta objetiva.

La ERS se puede realizar a todos los pacientes candidatos a neuromodulación de raíces sacras excepto a aquellos que sean portadores de marcapasos cardiaco (peligro de interferencias), los que precisen una Resonancia Nuclear Magnética durante el periodo de prueba (el campo magnético puede desplazar el electrodo e inducir estímulos no deseables), y en cuando no sean capaces de manejar adecuadamente el estimulador.

Las complicaciones descritas en la literatura son escasas. Así la serie de Schmidt y cols. ${ }^{10}$, con más de 1.200 exploraciones realizadas entre 1980 y 1997, no refiere ninguna complicación significativa, tan solo algunos pacientes notaron un cierto grado de dolor durante 2-3 días en el punto de la punción $\mathrm{y}$, ocasionalmente, una acentuación de los síntomas durante 1-2 días tras la retirada del electrodo temporal; además también describió algunos cambios temporales del hábito intestinal. Por ello concluye que es una exploración totalmente inocua y segura. Por otra parte, el estudio multicéntrico MDT-103 recoge los efectos adversos de 914 exploraciones realizadas en 581 pacientes; en el $18.2 \%$ del total de pruebas realizadas se produjo algún efecto adverso (Tabla IV), siendo el más frecuente la movilización del electrodo.

\section{TABLA IV}

EFECTOS ADVERSOS REGISTRADOS EN EL ESTUDIO MDT-103

\begin{tabular}{||l|c|}
\hline Efecto adverso & $\%$ \\
\hline Desplazamiento del electrodo & 11,8 \\
\hline $\begin{array}{l}\text { Desconexión del electrodo temporal } \\
\text { del estimulador externo }\end{array}$ & 2,6 \\
\hline Dolor temporal & 2,1 \\
\hline $\begin{array}{l}\text { Cambio en tránsito intestinal o función } \\
\text { de vaciado }\end{array}$ & 0,6 \\
\hline Infección o irritación de la piel & 0,6 \\
\hline
\end{tabular}


En nuestra serie no hemos tenido ninguna complicación significativa. Tan solo una paciente refirió molestias a nivel de la zona de implantación del electrodo que desaparecieron tras la retirada de éste, mientras que otra refirió dolor anal y debilidad intensa en la pierna ipsilateral a la raíz estimulada, desapareciendo los efectos secundarios tras la retirada del electrodo.

Los resultados positivos de la ERS varían ampliamente según las series (Tabla V), oscilando entre 24-75\%. Esta gran variabilidad hace sospechar que exista un elevado número de falsos negativos, principalmente relacionados con la movilización del electrodo $(20 \%)^{2}$. En nuestra serie la movilización precoz se produjo en 8 exploraciones $(18,6 \%)$ y al repetir la prueba ésta resultó positiva en 5; además 7 de las 8 movilizaciones se produjeron antes de utilizar el electrodo de nuevo diseño que minimiza el riesgo de movilización. Estos nuevos electrodos tienen una fijación pasiva suficiente que permite la implantación y fácil retirada pero, a la vez, mantienen su posición durante la fase subcrónica, incluso si ésta se prolonga más de 7 días ${ }^{11}$. También podemos obtener una prueba falsamente negativa en la fase aguda de la ERS si infiltramos anestésico directamente en el interior del foramen, ya que se abolen las respuestas motoras y sensitivas del estímulo eléctrico.

En función de la respuesta obtenida tras la estimulación temporal, se pueden plantear diversas actitudes. Si la respuesta es $>50 \%$, el paciente es inicialmente candidato al implante definitivo.
Si se produce cierto grado de mejoría pero es menor al 50\%, habitualmente planteamos una segunda exploración de raíces sacras para aclarar el efecto terapéutico de la técnica. Este es el motivo por el que repetimos algunas ERS en ciertos pacientes. Si no se produce ningún efecto terapéutico tras la estimulación temporal, existe la posibilidad de que nos encontremos ante un falso negativo. Para disminuir la incidencia de falsos negativos, existen distintas opciones: a) repetir la ERS; b) realizar una estimulación bilateral (doble electrodo sacro derecho e izquierdo); c) efectuar un implante en dos fases: se coloca quirúrgicamente el electrodo definitivo sacro y se conecta a un estimulador temporal externo durante 2 ó 3 semanas, tiempo suficiente para efectuar distintas programaciones de estimulación y valorar su eficacia terapéutica.

En el seguimiento a largo plazo de los enfermos tras el implante definitivo, aproximadamente hasta en un $25 \%$ desaparece el efecto terapéutico, por lo que solamente entre un $35-40 \%$ de los pacientes sometidos a ERS tendrán una respuesta positiva a largo plazo tras el implante ${ }^{12}$. La explicación más lógica de la falta de efecto terapéutico es la pérdida de contacto eficiente del electrodo con la raíz nerviosa, lo que conlleva la realización de un gran número de reintervenciones para reposicionar correctamente el electrodo definitivo.

En la actualidad el único factor predictivo de respuesta es la positividad de la ERS, no debiendo ofrecer el implante definitivo si no ha habido una mejoría significativa de los síntomas durante la

\section{TABLA V}

RESULTADOS DE LA ERS

\begin{tabular}{|c|c|c|c|c|}
\hline Grupo & $\begin{array}{c}\text { Grupo de } \\
\text { tratamiento }\end{array}$ & $\mathbf{n}$ & $\begin{array}{l}\text { PNE + } \\
\text { n (\%) }\end{array}$ & $\underset{n}{\text { Implantes }}$ \\
\hline Bosch y Groen & IUU & 85 & $46(54)$ & 45 \\
\hline Grünefald y cols. & Todos & 184 & $55(30)$ & 55 \\
\hline Weil y cols. & Todos & 100 & $36(36)$ & 36 \\
\hline Elabbady y cols. & DV+dolor pelviano & 50 & $17(34)$ & 14 \\
\hline Siegel & IUU & 49 & $18(37)$ & 12 \\
\hline Swinn y cols. & Sdr. Fowler & 30 & $21(70)$ & 11 \\
\hline Schmidt y cols. (MDT-103) & Todos & $581^{*}$ & $260(45)$ & 219 \\
\hline Total & & 1079 & $453(42)$ & 392 \\
\hline
\end{tabular}

*Sólo los datos de la IUU han sido publicados. 
$\mathrm{ERS}^{2}$. Así cuando existe algún tipo de duda sobre esta mejoría se puede realizar más de una ERS; algunos grupos realizan dos pruebas de forma sistemática ${ }^{13}$. También se puede realizar un implante en dos tiempos. Se ha propuesto también alargar el periodo de tiempo de la fase subcrónica y realizar un control urodinámico como prueba objetiva de mejoría ${ }^{14}$. Otros abogan por un abordaje más agresivo consistente en la implantación de un electrodo bilateral mediante la realización previa de una laminectomía sacra para así asegurar el contacto directo y estable de los electrodos junto a la raíz nerviosa, aunque con esto se aumenta el coste y la morbilidad del tratamiento ${ }^{15}$.

En los últimos años se ha desarrollado una técnica de implante del electrodo mínimamente invasiva, que podría cambiar la posición de la ERS como técnica imprescindible en la evaluación de los pacientes candidatos a neuromodulación sacra. La técnica desarrollada por Spinelli y cols. ${ }^{16}$ consiste en la implantación percutánea bajo anestesia local y control radiológico del electrodo definitivo a nivel de S3. El implante se realiza en dos fases: el paciente recibe el estímulo eléctrico durante un periodo de 2 semanas mediante un estimulador externo temporal, analizando la respuesta clínica y modificando los parámetros de estimulación si se considera necesario. Si la respuesta es adecuada, se procede al implante del generador de impulsos eléctricos definitivo. La analogía de esta técnica con la ERS convencional es evidente: se efectúa con anestesia local y tras comprobar las respuestas sensitivo-motoras adecuadas se pasa a una fase de estimulación temporal con un estimulador externo. Las ventajas de esta técnica son claras: estimulamos con el electrodo definitivo, que aporta una estimulación más eficiente y con la posibilidad de una programación más específica, disminuyendo la tasa de falsos negativos por estimulación inadecuada y por movilización. Además, se simplifica de forma significativa la técnica quirúrgica de implante del electrodo. Puede que en un futuro se utilice esta técnica para la selección de pacientes candidatos al implante de un marcapasos urinario definitivo. Sin embargo, actualmente la principal crítica a esta posibilidad es económica: el coste de un electrodo definitivo es mucho mayor que el de un electrodo temporal.
Así pues, actualmente la exploración de raíces sacras constituye una técnica diagnóstica fundamental para la selección de pacientes con DMC candidatos al implante de un estimulador sacro definitivo.

\section{REFERENCIAS}

1. DIJKEMA HE, WEIL EH, MIJS PT, JANKNEGT RA.: Neuromodulation of sacral nerves for incontinence and voiding dysfunctions. Clinical results and complications. Eur Urol 1993; 24: 72-76.

2. WEIL EH, RUIZ-CERDA JL, EERDMANS PH, JANKNEGT RA, VAN KERREBROECK PE.: Clinical results of sacral neuromodulation for chronic voiding dysfunction using unilateral sacral foramen electrodes. World J Urol 1998; 16: 313-321.

3. WEIL EH, RUIZ-CERDÁ JL, EERDMANS PH, JANKNEGT RA, BEMELMANS BLH, VAN KERREBROECK PE.: Sacral root neuromodulation in the treatment of refractory urinary urge incontinence: a prospective randomized clinical trial. Eur Urol 2000; 37: 161-171.

4. VAN KERREBROECK PE.: The role of electrical estimulation in voiding dysfunction. Eur Urol 1998; 34: 27-30.

5. BOSCH JLHR, GROEN J.: Sacral (S3) segmental nerve estimulation as a treatment for urge incontinence in patients with detrusor instability: results of chronic electrical stimulation using an implantable neural prosthesis. J Urol 1995, 154: 504-507.

6. SHAKER HS, HASSOUNA M.: Sacral nerve root neuromodulation. an effective treatment for refractory urge incontinence. J Urol 1998; 159: 1516-1519.

7. HASSOUNA MM, ELHILALI MM.: Role of the sacral roo $t$ stimulator in voiding dysfunction. preliminary report. World J Urol 1991; 9: 145-148.

8. THON WF, BASKIN LS, JONAS U, TANAGHO EA, SCHMIDT RA.: Surgical principles of sacral foramen electrode implantation. World $J$ Urol 1991; 9: 133-137.

9. THON WF, BASKIN LS, JONAS U, TANAGHO EA, SCHMIDT RA.: Neuromodulation of voiding dysfunction and pelvic pain. World $J$ Urol 1991; 9: 138-141.

10. SCHMIDT R, SENN E, TANAGHO E.: Functional evaluation of sacral nerve root integrity. Report of a technique. Urology 1990; 35: 388392.

11. WEIL EH, RUIZ-CERDÁ JL, VAN DEN BOGAARD AE, VAN KERREBROECK PhEV.: Novel test lead designs for sacral nerve stimulation: improved passive fixation in an animal model. J Urol 2000; 164: 551555.

12. BOSCH J.: Sacral neuromodulation in the treatment of the unstable bladder. Curr Opin Urol 1998; 8: 287-291.

13. CAPPELLANO F, CIOTTI MG, OIZZOCCARO M, CATANZARO M, SANTANBROGIO S, CATANZARO F.: Sacral root neuromodulation in the treatment of female urge and mixed urinary incontinence. Urogynaecologia IJ 1998; 12: 111-121.

14. EVERAERT K, PLANCKE H, LEFEVERE F, OOSTERLINCK W.: The urodynamic evaluation of neuromodulation in patients with voiding disorders. Brit J Urol 1997; 79: 702-707.

15. BRAUN PM, BOCHHERT J, BROSS S, SCHEEPE JR, ALKEN P, JUENEMANN P.: Tailored laminectomy: a new technique for neuromodulator implantation. J Urol 1999; 162: 1607-1609.

16. SPINELLI M, MAMO GA, ARDUINI A, GERBER M, GIARDIELLO G.: Evolution of a minimally-invasive procedure for sacral neuromodulation. En: Jonas U, Grünewald V, (eds). New Perspectives in Sacral Nerve Stimulation. Londres, Martin Dunitz, 2002: 217-222.

Dr. S. Arlandis Guzmán

C/ Mercat, $11-1^{a}$

46190 Riba-roja de Turia (Valencia)

(Trabajo recibido el 10 diciembre de 2002) 